\title{
Universal Ratios of the Renormalization Group
}

\author{
G. Mussardo \\ Universita' dell'Insubria, Como (Italy) \\ INFN, Sezione di Trieste (Italy)
}

ABSTRACT: The scaling form of the free-energy near a critical point allows for the definition of various universal ratios of thermodynamical amplitudes. Together with the critical exponents they characterize the universality classes and may be useful experimental quantities. We show how these universal quantities can be computed for a particular class of universality by using several Quantum Field Theory methods

\section{Introduction}

One of the most important successes of Quantum Field Theory (QFT) in recent years consists of the quantitative analysis of the universality classes of two-dimensional statistical mechanics models near their second order phase transition points. Right at criticality, where the correlation length $\xi$ diverges, Conformal Field Theory (CFT) allows the exact determination of the spectrum of anomalous dimensions, the structure constants of the OPE algebra, the multi-point correlation functions etc. [i.1. However, the CFT data do not exhaust all physical information relative to the phase transitions. From a theoretical point of view, a perturbation of the conformal action is required in order to investigate the space of the coupling constants and its topology consisting of the location of the fixed points and the Renormalization Group flows which connect them. This rich and interesting subject has been deeply investigated by several authors in the last ten years (see, for instance, [3.] and references therein for a review). From a practical point of view, one has to take into account that in a real sample, conformal invariance may be broken by the presence of impurities or, simply, by an imperfect fine-tuning of the experimental knobs. Hence, to control these effects it becomes important to study the dynamics of the systems slightly away from criticality, in particular their responses to external fields. The most ambitious goal would be the determination of both the scaling function which fixes the equa- tion of state and the off-critical correlators of the various order parameters. Although the exact determination of the equation of state of a given universality class may often be a difficult task, the scaling property alone of the free-energy is nevertheless sufficient to extract numerous predictions on universal combinations of critical amplitudes. As will be discussed below, these universal combinations are pure numbers which can be extremely useful for the experimental identification of the universality classes.

\section{Universal Amplitude Ratios}

Consider a statistical model with $n$ relevant fields $\varphi_{i}(x)$ at criticality. Near the critical point, its action can be parameterised as

$$
\mathcal{A}=\mathcal{A}_{C F T}+g_{i} \int \varphi_{i}(x) d^{D} x .
$$

The scaling property of the order parameters is encoded into the asymptotic form of their twopoint functions $\left\langle\varphi_{i}(x) \varphi_{i}(0)\right\rangle \simeq \frac{1}{|x|^{4 \Delta_{i}}}$ for $|x| \rightarrow 0$ so that $\Delta_{i}$ is identified with the conformal dimension of the fields. Correspondingly the conjugate coupling constants $g_{i}$ behave as $g_{i} \sim \Lambda^{D-2 \Delta_{i}}$, where $\Lambda$ is a mass scale. Therefore, away from criticality there will be generally a finite correlation length $\xi$ which in the thermodynamical limit scales as $\xi \sim a\left(K_{i} g_{i}\right)^{-\frac{1}{D-2 \Delta_{i}}}$, where $a \sim \Lambda^{-1}$ may be regarded as a microscopic length scale. The terms $K_{i}$ are non-universal metric factors which depend on the unit chosen for measuring 
the external sources $g_{i}$, alias on the particular realization selected for representing the universality class. In the presence of several deformations of the conformal action, the most general expression for the scaling form of the correlation length may be written as

$$
\xi=\xi_{i} \equiv a\left(K_{i} g_{i}\right)^{-\frac{1}{D-2 \Delta_{i}}} \mathcal{L}_{i}\left(\frac{K_{j} g_{j}}{\left(K_{i} g_{i}\right)^{\phi_{j i}}}\right),
$$

where $\phi_{j i} \equiv \frac{D-2 \Delta_{j}}{D-2 \Delta_{i}}$ are the so-called crossover exponents whereas $\mathcal{L}_{i}$ are universal homogeneous scaling functions of the ratios $\frac{K_{j} g_{j}}{\left(K_{i} g_{i}\right)^{\phi_{j i}}}$. There are of course several (but equivalent) ways of writing these scaling forms, depending on which coupling constant is selected as a prefactor. In the limit where $g_{l} \rightarrow 0(l \neq i)$ but $g_{i} \neq 0$, equation (2.2) becomes

$$
\xi_{i}=a \xi_{i}^{0} g_{i}^{-\frac{1}{D-2 \Delta_{i}}} \quad, \quad \xi_{i}^{0} \sim K_{i}^{-\frac{1}{D-2 \Delta_{i}}} .
$$

Consider now the free-energy $\hat{f}\left[g_{1}, \ldots, g_{n}\right]$. This is a dimensionless quantity defined by

$e^{-\hat{f}\left(g_{1}, \ldots, g_{n}\right)}=\int \mathcal{D} \phi e^{-\left[\mathcal{A}_{C F T}+\sum_{i=1}^{n} g_{i} \int \varphi_{i}(x) d^{D} x\right]}$.

Assuming the validity of the hyperscaling hypothesis, in the thermodynamical limit its singular part (per unit of volume) will be proportional to the $D$ power of the correlation length. Let us denote the singular part of the free-energy for unit volume by $f\left[g_{1}, \ldots, g_{n}\right]$. Depending on which scaling form is adopted for the correlation length, we have correspondingly several (but equivalent) ways of parameterizing this quantity

$$
f_{i}\left[g_{1}, \ldots, g_{n}\right] \equiv\left(K_{i} g_{i}\right)^{\frac{D}{D-2 \Delta_{i}}} \mathcal{F}_{i}\left(\frac{K_{j} g_{j}}{\left(K_{i} g_{i}\right)^{\phi_{j i}}}\right) \text {. }
$$

The functions $\mathcal{F}_{i}$ are universal homogeneous scaling functions of the ratios $\frac{K_{j} g_{j}}{\left(K_{i} g_{i}\right)^{\phi_{j i}}}$.

Let us consider now the definition of the thermodynamical quantities related to the various derivatives of the free-energy. We will adopt the notation $\langle\ldots\rangle_{i}$ to denote expectation values computed in the off-critical theory obtained by keeping (at the end) only the coupling constant $g_{i}$ different from zero. The first quantities to consider are the vacuum expectation values (VEV) of the fields $\varphi_{j}$ which can be parameterized as

$$
\left\langle\varphi_{j}\right\rangle_{i}=-\left.\frac{\partial f_{i}}{\partial g_{j}}\right|_{g_{l}=0} \equiv B_{j i} g_{i}^{\frac{2 \Delta_{j}}{D-2 \Delta_{i}}},
$$

with

$$
B_{j i} \sim K_{j} K_{i}^{\frac{2 \Delta_{j}}{D-2 \Delta_{i}}}
$$

The above relations can be equivalently expressed as

$$
g_{i}=D_{i j}\left(\left\langle\varphi_{j}\right\rangle_{i}\right)^{\frac{D-2 \Delta_{i}}{2 \Delta_{j}}},
$$

with

$$
D_{i j} \sim \frac{1}{K_{i} K_{j}^{\frac{D-2 \Delta_{i}}{2 \Delta_{j}}}}
$$

The generalized susceptibilities of the model are defined by

$$
\hat{\Gamma}_{j k}^{i}=\frac{\partial}{\partial g_{k}}\left\langle\varphi_{j}\right\rangle_{i}=-\left.\frac{\partial^{2} f_{i}}{\partial g_{k} \partial g_{j}}\right|_{g_{l}=0} .
$$

They are obviously symmetrical in the two lower indices. By extracting their dependence on the coupling constant $g_{i}$, they can be expressed as

$$
\hat{\Gamma}_{j k}^{i}=\Gamma_{j k}^{i} g_{i}^{\frac{2 \Delta_{j}+2 \Delta_{k}-D}{D-2 \Delta_{i}}}
$$

with

$$
\Gamma_{j k}^{i} \sim K_{j} K_{k} K_{i}^{\frac{2 \Delta_{j}+2 \Delta_{k}-D}{D-2 \Delta_{i}}}
$$

The various quantities obtained by taking the derivatives of the free-energy obviously contain metric factors (the quantities $K_{i}$ ) which make their values not universal. However, it is always possible to consider special combinations thereof in such a way to cancel out all metric factors. Here we propose the consideration of the following universal ratios

$$
\begin{gathered}
\left(R_{c}\right)_{j k}^{i}=\frac{\Gamma_{i i}^{i} \Gamma_{j k}^{i}}{B_{j i} B_{k i}} ; \\
\left(R_{\chi}\right)_{j}^{i}=\Gamma_{j j}^{i} D_{j j} B_{j i}^{\frac{D-4 \Delta_{j}}{2 \Delta_{j}}} ; \\
R_{\xi}^{i}=\left(\Gamma_{i i}^{i}\right)^{1 / D} \xi_{i}^{0} ; \\
\left(R_{A}\right)_{j}^{i}=\Gamma_{j j}^{i} D_{i i}^{\frac{4 \Delta_{j}+2 \Delta_{i}-2 D}{D-2 \Delta_{i}}} B_{i j}^{\frac{2 \Delta_{j}-D}{\Delta_{i}}} ; \\
\left(Q_{2}\right)_{j k}^{i}=\frac{\Gamma_{j j}^{i}}{\Gamma_{j j}^{k}}\left(\frac{\xi_{k}^{0}}{\xi_{j}^{0}}\right)^{D-4 \Delta_{j}} ;
\end{gathered}
$$

From their definition, these quantities are pure numbers attached to the universality classes and can be used to characterize them. In fact, the amplitude ratios are numbers which typically present significant variations between different classes of 
universality, whereas the critical exponents usually assume small values which only vary by a small percent by changing the universality classes. Hence the universal ratios may be ideal marks of the critical scaling regime [4]. It is also worth emphasizing that, from an experimental point of view, it should be simpler to measure universal amplitude ratios rather than critical exponents: to determine the former quantities one needs to perform several measurements at a single, fixed value of the coupling which drives the system away from criticality whereas to determine the latter, one needs to make measurements over several decades along the axes of the off-critical couplings. Moreover, although not all of them are independent, the universal ratios are a larger set of numbers than the critical exponents and therefore permit a more precise determination of the class of universality.

\section{Tricritical Ising Model}

The universal ratios of the two-dimensional Tricritical Ising Model (TIM) were computed in refs. [ i. tailed discussion of their evaluation. There are several reasons for considering the class of universality associated to this model. First of all, from the experimental point of view, a number of physical systems exhibit a tricritical Ising behavior, among them fluid mixtures, metamagnets or order-disorder transitions in absorbed systems [i, (for a review on the theory of tricritical points, see

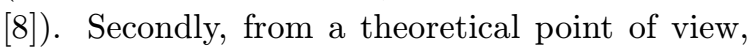
the TIM is still sufficiently simple to be solved but at the same time it presents an extremely rich and fascinating structure of excitations away from criticality. In a Landau-Ginzburg approach, the TIM is associated to a $\Phi^{6}$-theory near its tricritical point $[\overline{9}]$, although this approach is often too elementary for the understanding of some of its remarkable symmetries. Depending on the direction in the phase space in which the system is moved away from criticality, one can observe, for instance, a behavior ruled by the exceptional root system $E_{7}\left[\begin{array}{l}{[1} \\ 2\end{array}\right.$

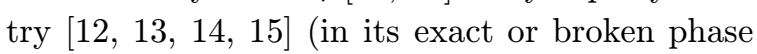
realization) or by an asymmetrical pair of kinks $\left[1 \overline{1} \bar{\sigma}_{1}^{\prime}, 1 \bar{z}_{1}, 1\right]$ low-temperature phase is easily obtained from the one of its high-temperature phase because of the self-duality of the model.

A convenient way to determine the universal ratios of the TIM consists of adopting a Quantum Field Theory (QFT) approach, as it was done in the original references $[5,5,6$. It would be obviously interesting to compute and to compare them by using a lattice formulation as, for instance, the one studied in [19 19.' In a QFT approach, one takes initially advantage of the exact solution of the model at criticality. The bidimensional TIM is described by the second representative of the unitary series of minimal models of CFT [i1, , is equal to $c=\frac{7}{10}$ and the exact conformal weights of the scaling fields are given by

$$
\Delta_{l, k}=\frac{(5 l-4 k)^{2}-1}{80} \quad, \quad \begin{aligned}
& 1 \leq l \leq 3 \\
& 1 \leq k \leq 4
\end{aligned}
$$

There are six primary scalar fields $\phi_{\Delta, \bar{\Delta}}$, which close an algebra under the Operator Product Expansion

$$
\begin{aligned}
& \phi_{i}\left(z_{1}, \bar{z}_{1}\right) \phi_{j}\left(z_{2}, \bar{z}_{2}\right) \sim \\
& \left.\sim \sum_{k} c_{i j k}\left|z_{1}-z_{2}\right|^{-2\left(\Delta_{i}+\Delta_{j}-\Delta_{k}\right)} \phi_{k}\left(z_{2}, \bar{z}_{2}\right) .2\right)
\end{aligned}
$$

The skeleton form of this OPE algebra and the relative structure constants of the Fusion Rules of the TIM were given in ref. [i $\left[\overline{6}^{\prime}\right]$. The six primary fields can be identified with the normal ordered composite LG fields $[\overline{9} \overline{0}]$. With respect to their properties under the $Z_{2}$ spin-reversal transformation $Q: \Phi \rightarrow-\Phi$ we have:

1. two odd fields: the leading magnetization operator $\sigma=\phi_{\frac{3}{80}, \frac{3}{80}} \equiv \Phi$ and the subleading magnetization operator $\sigma^{\prime}=\phi_{\frac{7}{16}, \frac{7}{16}} \equiv: \Phi^{3}$ :

2. four even fields: the identity operator $1=$ $\phi_{0,0}$, the leading energy density $\varepsilon=\phi_{\frac{1}{10}, \frac{1}{10}} \equiv$ : $\Phi^{2}$ :, the subleading energy density $t=\phi_{\frac{6}{10}, \frac{6}{10}} \equiv$ : $\Phi^{4}$ :, which in metamagnets assumes the meaning of the density of the annealed vacancies, and the field $\varepsilon "=\phi_{\frac{3}{3}, \frac{3}{2}}$. The OPE of the even fields form a subalgebra of the Fusion Rules.

In the TIM there is another $Z_{2}$ transformation the Kramers-Wannier duality $D$ - under which the fields transform as follows: 
- the order magnetization operators are mapped onto their corresponding disorder operators

$$
\mu=D^{-1} \sigma D \quad, \quad \mu^{\prime}=D^{-1} \sigma^{\prime} D .
$$

- the even fields are mapped onto themselves,

$$
D^{-1} \varepsilon D=-\varepsilon \quad, \quad D^{-1} t D=t \quad,
$$

Given the $Z_{2}$-spin odd parity of the two magnetic fields, the off-critical theories obtained by their perturbation are independent of the sign of their associate couplings. The odd parity of the energy field under the other $Z_{2}$ symmetry is responsable for the duality mapping between the high and low temperature phases. On the other hand, two different physical situations arise depending on the sign of the coupling relative to the perturbation of the vacancy density field $t(x)$. It is important to notice that, excluding the leading magnetic perturbation, all the others give rise to integrable QFT away from criticality. To simplify the formulae below, it is convenient to adopt the compact notation $\varphi_{i}(i=1,2,3,4)$ to denote collectively all these fields, so that $\varphi_{1}=\sigma, \varphi_{2}=\varepsilon, \varphi_{3}=\sigma^{\prime}$ and $\varphi_{4}=t$. Let us discuss now some aspects of QFT which make possible the determination of the universal ratios and the strategy we have used to achieve these results.

\section{Quantum Field Theory Approach}

Each coupling constant $g_{i}(i=1, \ldots, 4)$ relative to the relevant operator $\varphi_{i}(x)$ of the TIM is a dimensional quantity which can be related to the lowest mass-gap $m_{i}=\xi_{i}^{-1}$ of the off-critical theory according to the formula

$$
m_{i}=\mathcal{C}_{i} g_{i}^{\frac{1}{2-2 \Delta_{i}}} .
$$

When the QFT associated to the action (2.1יi) is integrable, the pure number $\mathcal{C}_{i}$ can be exactly determined by means of the Thermodynamical Bethe Ansatz $\left[2 \overline{2}, 1, \overline{2} \overline{1}_{-1}^{\prime}\right]$. When the theory is not integrable (this is the case for the magnetic deformation of the TIM), the constant $\mathcal{C}_{i}$ can be nevertheless determined by a numerical method, based on the socalled Truncated Conformal Space Approach [202i]. In conclusion, for all individual deformations of the TIM we are able to completely set the relationship which links the coupling constant to the mass-gap of the theory and therefore to switch freely between these two variables.

Another set of quantities which can be fixed by QFT are the matrix elements of the order parameters, the simplest ones being the vacuum expectation values (VEV). In this case we have

$$
\left\langle\varphi_{j}\right\rangle_{i}=B_{j i} g_{i}^{\frac{\Delta_{j}}{1-\Delta_{i}}} .
$$

When the theory is integrable, the constant $B_{j i}$ can be fixed exactly, thanks to the results of a remarkable series of papers $\left[2 \overline{3}^{1}, 2 \overline{2} \overline{4}_{1}^{n}\right.$. When it is not integrable, the constant $B_{j i}$ can be nevertheless estimated by means of a numerical approach, as firstly shown in $\overline{2} \overline{5}$. Hence, also in this case, we are able to determine completely these quantities. Moreover, as shown in [i, tion of the numerical approach of ref. [2 $\overline{2} \bar{n}]$ often leads to a reasonable estimation of the matrix elements of the order parameters between the vacuum states and some of the excited states, as for instance $\left\langle 0\left|\varphi_{j}\right| A_{k}\right\rangle_{i}$ where $A_{k}$ is a one-particle state of mass $M_{k}$. These quantities turn out to be useful for obtaining sensible approximation of the large-distance behavior of several correlators.

Another useful piece of information on the offcritical dynamics can be obtained by exploiting the properties of the stress-energy tensor $T_{\mu \nu}(x)$. In the presence of the perturbing field $\varphi_{i}$, the trace of the stress-energy tensor is different from zero and can be expressed as

$$
\Theta(x)=2 \pi g_{i}\left(2-2 \Delta_{i}\right) \varphi_{i} .
$$

The trace of the stress-energy tensor enters a useful sum rule - called the $\Delta$-theorem sum rule [3i - which reads

$$
\Delta_{j}=-\frac{1}{4 \pi\left\langle\varphi_{j}\right\rangle_{i}} \int d^{2} x\left\langle\Theta(x) \varphi_{j}(0)\right\rangle_{i}^{c},
$$

i.e. it relates the conformal dimension $\Delta_{j}$ of the field $\varphi_{j}$ to its VEV and to the integral of its connected off-critical correlator with $\Theta(x)$. It is easy to see that the above formula simply expresses the content of the fluctuation-dissipation theorem and when the above integral diverges, so does the VEV in the denominator, in such a way that eq. (4.4) always keeps its validity [i].

Basic quantities in the universal ratios are the generalized susceptibilities $\Gamma_{j k}^{i}$ which, by using the 
fluctuation-dissipation theorem, can be expressed as

$$
\hat{\Gamma}_{j k}^{i}=\int d^{2} x\left\langle\varphi_{j}(x) \varphi_{k}(0)\right\rangle_{c}^{i}
$$

By extracting their dependence on the coupling constant $g_{i}$, we have $\hat{\Gamma}_{j k}^{i}=\Gamma_{j k}^{i} g_{i}^{\frac{\Delta_{j}+\Delta_{k}-1}{1-\Delta_{i}}}$ with

$$
\Gamma_{j k}^{i}=\mathcal{C}_{i}^{2 \Delta_{j}+2 \Delta_{k}-2} \int d \tau \frac{1}{\tau^{2 \Delta_{j}+2 \Delta_{k}}} Q_{j k}(\tau) .
$$

Some of the above susceptibilities can be determined exactly, such as the components $\Gamma_{i k}^{i}$, whose values are provided by the $\Delta$-theorem sum rule

$$
\Gamma_{i k}^{i}=-\frac{\Delta_{k}}{1-\Delta_{k}} B_{k i} .
$$

In all other cases, when an exact formula is not available, the strategy to evaluate the generalized susceptibilities relies on two different representations of the correlators. These representations have the advantage to converge very fast in two distinct regions.

The first representation is based on Conformal Perturbation Theory (with the employment of the non-analytic expression of the VEV) [32 approach the two-point correlators are expressed as

$$
\left\langle\varphi_{i}(x) \varphi_{j}(0)\right\rangle=\sum_{i} C_{i j}^{p}(g ; x)\left\langle A_{p}(0)\right\rangle
$$

where the off-critical structure constants $C_{j k}^{p}(g ; x)$ admit the expansion

$$
C_{i j}^{p}(g ; x)=r^{2\left(\Delta_{p}-\Delta_{i}-\Delta_{j}\right)} \sum_{n=0}^{\infty} C_{i, j}^{p(n)}\left(g r^{2-2 \Delta_{\Phi}}\right)^{n},
$$

$(r=|x|)$ and can be computed perturbatively in $g$. Their first order contribution is given by [3.

$$
C_{i, j}^{p(1)}=-\int^{\prime} d^{2} w\left\langle\tilde{A}^{p}(\infty) \tilde{\Phi}(w) \tilde{\varphi}_{i}(1) \tilde{\varphi}_{j}(0)\right\rangle_{C F T},
$$

where the prime indicates a suitable infrared (large distance) regularization of the integral. This representation allows a very efficient estimation of the correlation function in its short distance regime $r \ll \xi$.

The second representation is based on the Form Factors and allows an efficient control of its large distance behavior, i.e. when $r \gg \xi$. In this second representation, one makes use of the knowledge of the off-critical mass spectrum of the theory to express the correlators as

$$
\left\langle\varphi_{i}(x) \varphi_{j}(0)\right\rangle=\sum_{n=0}^{\infty} g_{n}(r)
$$

where

$$
\begin{aligned}
& g_{n}(r)=\int_{\theta_{1}>\theta_{2} \ldots>\theta_{n}} \frac{d \theta_{1}}{2 \pi} \ldots \frac{d \theta_{n}}{2 \pi} e^{-r \sum_{k=1}^{n} m_{k} \cosh \theta_{k}} \\
& \times\left\langle 0\left|\varphi_{i}(0)\right| \ldots A_{a_{n}}\left(\theta_{n}\right)\right\rangle\left\langle\ldots A_{a_{n}}\left(\theta_{n}\right)\left|\varphi_{j}(0)\right| 0\right\rangle .
\end{aligned}
$$

$\left|A_{a_{1}}\left(\theta_{1}\right) \ldots A_{a_{n}}\left(\theta_{n}\right)\right\rangle$ are the multi-particle states relative to the excitations of mass $m_{k}$, with relativistic dispersion relations given by $E=m_{k} \cosh \theta$, $p=m_{k} \sinh \theta$, where $\theta$ is the rapidity variable. The spectral representation ( $\overline{4} . \overline{1} \overline{2})$ is obviously an expansion in the parameter $e^{-\frac{r^{-}}{\xi}}$, where $\xi^{-1}=m_{1}$ is the lowest mass-gap.

Basic quantities of the large distance approach are the Form Factors (FF), i.e. the matrix elements of the operators $\varphi_{i}$ on the physical asymptotic states $\left[26, \overline{6}, \overline{2} \overline{7}_{1}\right]$

$F_{a_{1}, \ldots, a_{n}}^{\varphi_{i}}\left(\theta_{1}, \ldots, \theta_{n}\right)=\left\langle 0\left|\varphi_{i}(0)\right| A_{a_{1}}\left(\theta_{1}\right) \ldots A_{a_{n}}\left(\theta_{n}\right)\right\rangle$.

It is worth emphasizing that the above quantities are unaffected by renormalization effects since physical excitations are employed in their definitions. For scalar operators, relativistic invariance requires that the FF only depend on the rapidity differences $\theta_{i}-\theta_{j}$. In our calculation we have only insert into the spectral representations the one-particle and two-particle FF, computed according to the analysis of ref. [2 $\left.28^{\prime \prime}, 29\right]$.

Both the representations $(4.8)$ and $\left(\begin{array}{l}1 \\ 4\end{array} . \overline{1} \overline{1}_{1}^{1}\right)$ are known to converge very fast (see, for instance [30], 32.] and therefore they are efficiently approximated by their lowest terms, which therefore can be evaluated with a relatively little analytical effort. These considerations obviously lead to the estimation of the integral (4.5) according to the following steps:

1. Express the integral in polar coordinates as

$$
\hat{\Gamma}_{j k}^{i}=2 \pi \int_{0}^{+\infty} d r r\left\langle\varphi_{j}(r) \varphi_{k}(0)\right\rangle_{c}^{i}
$$

and split the radial integral into two pieces as

$$
I=\int_{0}^{+\infty} d r r\left\langle\varphi_{j}(r) \varphi_{k}(0)\right\rangle_{c}^{i}
$$




$$
\begin{aligned}
& =\int_{0}^{R} d r r\langle\ldots\rangle_{c}^{i}+\int_{R}^{+\infty} d r r\langle\ldots\rangle_{c}^{i} \\
& \equiv I_{1}(R)+I_{2}(R) .
\end{aligned}
$$

2. Use the best available short-distance representation of the correlator to evaluate $I_{1}(R)$ as well as the best available estimate of its large-distance representation to evaluate $I_{2}(R)$.

3. Optimize the choice of the parameter $R$ in such a way to obtain the best evaluation of the whole integral. In practice, this means looking at that value of $R$ for which a plateau is obtained for the sum of $I_{1}(R)$ and $I_{2}(R)$. Say in another way, $R$ belongs to that interval where there is an overlap between the short-distance and the long-distance expansion of the correlator (see, for instance, Figure 1 , relative to the correlator $\left\langle\varphi_{1}(x) \varphi_{1}(0)\right\rangle_{1}$ in the high-temperature phase).

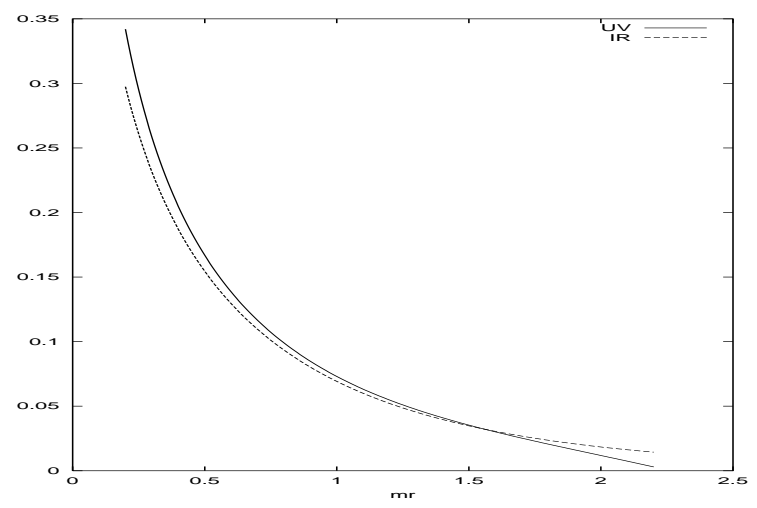

Figure 1: Continuous line $=U \mathrm{UV}$ approximation, dashed line $=$ IR approximation. An overlap of the curves is observed around $m r \sim 1$.

Gathering all the results relative to the above quantities, a set of universal ratios for the TIM have been obtained. Some of them are exact, like $\left(R_{c}\right)_{1, k}^{1}=$ $\frac{240}{5929} \Delta_{k},\left(R_{c}\right)_{2, k}^{2}=\frac{10}{81} \Delta_{k}(k=1, \ldots, 4)$. Those relative to the low and high temperature phase of the model denoted by an upper index ₹ respectively, are in Table 1. An interesting universal ratio is provided in this case by the correlation lengths $\xi^{ \pm}$, measured at the same displacement above and below the critical temperature (as extracted from the correlation function of the magnetic operator using its duality properties)

$$
\frac{\xi^{+}}{\xi^{-}}=2 \cos \left(\frac{5 \pi}{18}\right) \approx 1.28557 \ldots
$$

which can be inferred by the exact mass spectrum of the model and the parity properties of the ex-

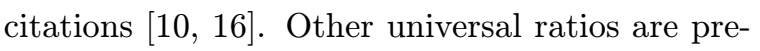
sented in Tables $2-5$ and a more complete set of

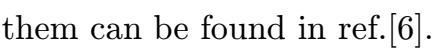

In view of the predictivity showed by the theoretical approach, it would be interesting to have their experimental confirmation. It would be equally useful to apply the methods discussed here to other models in such a way to bridge a closer contact between theoretical and experimental results in twodimensional physics.

\section{Acknowledgments}

This work was supported by the TMR Network "Integrability, non-perturbative Effects and Symmetry in Quantum Field Theory', contract number FMRX-CT96-0012.

\section{References}

[1] A.A. Belavin, A.M. Polyakov and A.B. Zamolodchikov, Nucl. Phys. B 241 (1994), 333.

[2] D. Friedan, Z. Qiu, S. Shenker, Phys. Rev. Lett. 52 (1984), 1575

[3] G. Mussardo, Phys. Rep. 218 (1992), 215.

[4] V. Privman, P.C. Hohenberg and A. Aharony, Universal Critical-Point Amplitude Relations, in Phase Transitions and Critical Phenomena, edited by C. Domb and J.L. Lebowitz (Academic Press Limited, London, 1991), Vol. 14.

[5] D. Fioravanti, G. Mussardo and P. Simon, Phys. Rev. Lett. 85 (2000), 126.

[6] D. Fioravanti, G. Mussardo and P. Simon, Universal Amplitude Ratios of the Renormalization Group: Two-dimensional Tricritical Ising Model, cond-mat/0008216, to be published on Phys. Rev. E.

[7] M.J.Tejwani, O. Ferreira, O.E. Vilches, Phys. Rev. Lett. 44 (1980), 152

[8] I.D. Lawrie, S. Sarbach, in Phase Transitions and Critical Phenomena, vol. 9, C. Domb and J. Lebowitz (Eds.), New York (Academic Press 1984) 
[9] A.B. Zamolodchikov, Sov. J. Nucl. Phys. 44 (1986), 529.

[10] P. Christe, G. Mussardo, Nucl. Phys. B 330 (1990), 465.

[11] V.A. Fateev, A.B. Zamolodchikov, Int. J. Mod. Phys. A 5 (1990), 1025.

[12] D.A. Kastor, E.J. Martinec, S.H. Shenker, Nucl. Phys. B 316 (1989), 590

[13] Al.B. Zamolodchikov, Nucl. Phys. B 358 (1991), 524.

[14] G. Delfino, G. Mussardo and P. Simonetti, Phys. Rev. D 51 (1995), 6620.

[15] A.B. Zamolodchikov, in Beijing 1989, Proceedings Fields, strings and quantum gravity 349. Al.B. Zamolodchikov, Nucl. Phys. B 358 (1991), 497.

[16] M. Lässig, G. Mussardo and J.L. Cardy, Nucl. Phys. B 348 (1991), 591.

[17] F.A. Smirnov, Int. J. Mod. Phys. A 6 (1991), 1407.

[18] F. Colomo, A. Koubek and G. Mussardo, Int. J. Mod. Phys. A 7 (1992), 5281.

[19] S.O. Warnaar, B. Nienhuis and K.A. Seaton, Phys. Rev. Lett. 69 (1992), 710; S.O. Warnaar and P.A. Pearce, Int. J. Mod. Phys. A 11 (1996), 291; M.T. Batchelor and K.A. Seaton, Eur. Phys. J. B 5 (1998), 719; J. Suzuki, hep-th/9911216.

[20] Al.B. Zamolodchikov, Nucl. Phys. B 342 (1990), 695.

[21] V.A. Fateev, Phys. Lett. B 324 (1994), 45.

[22] V.P. Yurov and Al.B. Zamolodchikov, Int. J. Mod. Phys. A 6 (1991), 4557.

[23] S.Lukyanov and A.B. Zamolodchikov, Nucl. Phys. B 493 (1997), 571.

[24] V. Fateev, S. Lukyanov, A.B. Zamolodchikov, Al.B. Zamolodchikov, Nucl. Phys. B 516 (1998), 652.

[25] R. Guida and N. Magnoli, Phys. Lett. B 411 (1997) 127.

[26] M. Karowski, P. Weisz, Nucl. Phys. B 139 (1978), 445.

[27] F.A. Smirnov, Form Factors in Completely Integrable Models of Quantum Field Theory (World Scientific), 1992 and references therein.

[28] G. Delfino and G. Mussardo, Nucl. Phys. B 455 (1995), 724

[29] C. Acerbi, G. Mussardo and A. Valleriani, Int. J. Mod. Phys. A 11 (1996) 5327.
[30] J.L. Cardy and G. Mussardo, Nucl. Phys. B 410 (1993), 451.

[31] G. Delfino, P. Simonetti and J.L. Cardy, Phys. Lett. B 387 (1996), 327.

[32] Al.B. Zamolodchikov, Nucl. Phys. B 348 (1991), 619.

Table 1: Amplitude ratios $R_{j k}^{2}=\frac{\Gamma_{j k}^{2+}}{\Gamma_{j k}^{2-}}$.

\begin{tabular}{|ccc||ccc|}
\hline$R_{11}^{2}$ & $=$ & 3.54 & $R_{13}^{2}$ & $=$ & -2.06 \\
\hline$R_{22}^{2}$ & $=$ & 1 & $R_{24}^{2}$ & $=$ & -1 \\
\hline$R_{33}^{2}$ & $=$ & 1.30 & $R_{44}^{2}$ & $=$ & 1 \\
\hline
\end{tabular}

Table 2: Universal ratios $\left(R_{c}\right)_{j k}^{1}$ and $\left(R_{c}\right)_{j k}^{2-}$.

\begin{tabular}{|lll||lll|}
\hline$\left(R_{c}\right)_{22}^{1}=1.0510^{-2}$ & $\left(R_{c}\right)_{23}^{1}=4.8510^{-2}$ \\
\hline$\left(R_{c}\right)_{24}^{1}=6.710^{-2}$ & $\left(R_{c}\right)_{33}^{1}=3.810^{-1}$ \\
\hline \hline$\left(R_{c}\right)_{11}^{2-}=2.010^{-3}$ & $\left(R_{c}\right)_{14}^{2-}$ & $=$ & $-2.3410^{-2}$ \\
\hline$\left(R_{c}\right)_{13}^{2-}=1.7910^{-2}$ & $\left(R_{c}\right)_{33}^{2-}$ & $=$ & $3.410^{-1}$ \\
\hline
\end{tabular}

Table 3: Universal ratio $\left(R_{\chi}\right)_{j}^{i}$ for $i, j=1,2$.

\begin{tabular}{|cl||lll|}
\hline$\left(R_{\chi}\right)_{1}^{1}=3.89710^{-2}$ & $\left(R_{\chi}\right)_{2}^{2+}=0.1111$ \\
\hline$\left(R_{\chi}\right)_{2}^{1}=0.116$ & $\left(R_{\chi}\right)_{1}^{2-}=0.040$ \\
\hline$\left(R_{\chi}\right)_{1}^{2+}=0$ & $\left(R_{\chi}\right)_{2}^{2-}=0.1111$ \\
\hline
\end{tabular}

Table 4: Universal ratios $R_{\xi}^{i}$ and $\left(R_{A}\right)_{j}^{i}$ for $i, j=$ $1,2^{-}, 2^{+}$.

\begin{tabular}{|ccl||ccc|}
\hline$R_{\xi}^{1}$ & $=7.55710^{-2}$ & & & \\
\hline$R_{\xi}^{2+}$ & $=$ & $1.078410^{-1}$ & $R_{\xi}^{2-}$ & $=$ & $8.38910^{-1}$ \\
\hline \hline$\left(R_{A}\right)_{2+}^{1}$ & $=$ & 0 & $\left(R_{A}\right)_{2-}^{1}$ & $=$ & $3.91810^{-2}$ \\
\hline$\left(R_{A}\right)_{1}^{2+}$ & $=2.95810^{-1}$ & $\left(R_{A}\right)_{1}^{2-}$ & $=$ & $8.26010^{-1}$ \\
\hline
\end{tabular}

Table 5: Universal ratios $\left(Q_{2}\right)_{j k}^{i}$ for $i, j, k=1,2^{+}, 2^{-}$.

\begin{tabular}{|ccc||ccc|}
\hline$\left(Q_{2}\right)_{2^{+1}}^{1}$ & $=1.260$ & $\left(Q_{2}\right)_{2^{-1}}^{1}$ & $=$ & 1.884 \\
\hline$\left(Q_{2}\right)_{2^{+} 2^{+}}^{1}$ & $=$ & 1.973 & $\left(Q_{2}\right)_{2^{+} 2^{-}}^{1}$ & $=$ & 1.320 \\
\hline$\left(Q_{2}\right)_{11}^{2+}$ & $=$ & 1.56 & $\left(Q_{2}\right)_{11}^{2-}$ & $=$ & 0.442 \\
\hline$\left(Q_{2}\right)_{12^{-}}^{2+}$ & $=$ & 1.70 & & & \\
\hline
\end{tabular}

